\title{
Invasive fungal infections in critically-ill patients: A literature review and position statement from the IFI-clinical forum, Shiraz, Iran
}

\author{
Farid Zand ${ }^{1}$, Mohsen Moghaddami ${ }^{2}$, Mohammad Ali Davarpanah ${ }^{3}$, Mansoor Masjedi ${ }^{1}$, Reza \\ Nikandish1, Ali Amanati ${ }^{4}$, Mohammad Afarid ${ }^{5}$, Leila Nafarieh ${ }^{5}$ and Mohammad Nami ${ }^{5,6 *}$ \\ ${ }^{1}$ Shiraz Anesthesiology and Critical Care Research Center, Shiraz University of Medical Sciences, Shiraz, Iran \\ ${ }^{2}$ Non-Communicable Diseases Research Center, Shiraz University of Medical Sciences, Shiraz, Iran \\ ${ }^{3}$ HIV/AIDS Research Center, Shiraz University of Medical Sciences,Shiraz, Iran \\ ${ }^{4}$ Department of Pediatrics, Shiraz University of Medical Sciences, Shiraz, Iran \\ ${ }^{5}$ Behestan Medical Scientific Committee, Behestan Group, Tehran, Iran \\ ${ }^{6}$ Department of Neuroscience, School of Advanced Medical Sciences and Technologies, Shiraz University of \\ Medical Sciences, Shiraz, Iran \\ ${ }^{\Delta}$ The list of collaborators and consultants at IFI-CF, Shiraz, can be found in the appendix at the end of this report.
}

\begin{abstract}
Invasive fungal infections (IFIs) have increasingly been recognized as a serious clinical concern in critically-ill patients admitted to the intensive care units (ICUs). The most abundant pathogens in this population are Candida species. As such, a clear understanding on the epidemiology of this infection seems to be a key step in providing appropriate treatment. Expert input forums are among the practical approaches to define locally-adapted clinicalpathways with regard to debated medical perspectives. To agree upon a shared approach towards IFI management in ICU, an interdisciplinary panel of experts from infectious diseases and intensive care fields met up in Shiraz on 28 November 2015 within the IFI-Clinical Forum (IFI-CF).This clinical forum aimed to view the available evidence, taking into consideration the recent practice guidelines on IFIs management in the ICU to arrive at an agreed position in current clinical practice. The aim of this summary is to discuss IFIs in ICU from epidemiology, the range of pathophysiology from colonization to the invasive infections, risk prediction, diagnosis and treatment perspectives.
\end{abstract}

KEY WORDS: INVASIVE CANDIDIASIS; INVASIVE FUNGAL INFECTIONS; CRITICAL CARE; CLINICAL PATHWAY

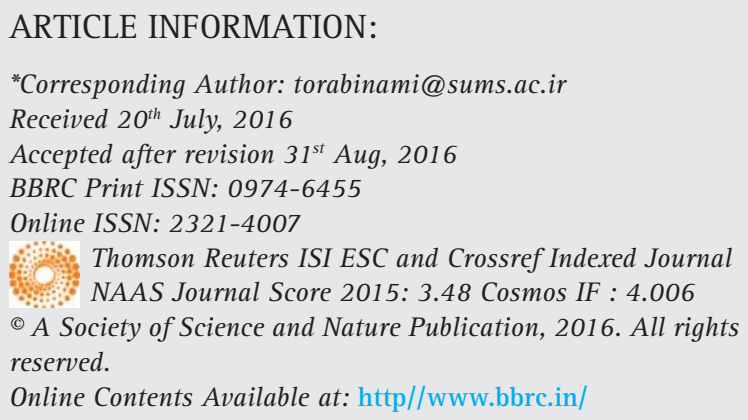




\section{INTRODUCTION}

With all the advancing medical care over the past decade, an increasing number immune-compromised patients have received in-hospital care and criticallyill patients have often experienced prolonged hospital stays. This might have potentially contributed to the development of invasive fungal infections (IFIs) resulting in a nearly five-fold increase in the incidence of such a clinical challenge in the past 10 years mainly in critical care setting(Elhoufi et al., 2014; Lepak \& Andes, 2011; Montagna et al., 2013). Despite all improved diagnostic methods, timely and specific diagnosis of IFIs in the ICUhas remained an uphillchallenge (Ahmadi et al., 2014; Avcu et al., 2016; He et al., 2015; Liu et al., 2015; Shi et al., 2015; Solmaz et al., 2016; Ullmann et al., 2012; Wu et al., 2016).

The reason for high mortality and morbidity of IFIs is at least two-fold. Firstly, the clinicians defer early empirical interventions as they may fail to consider IFIs and their possible outcome, and secondly, the etiology of infection is almost never adequately-established in time (Kollef, Micek, Hampton, Doherty, \&t Kumar, 2012; Pfaller \&t Diekema, 2007). To lessen such a burden, a clinical guidance on how to suspect, diagnose and treat IFIs and mainly invasive candidiasis (IC), which is the most frequent case in critical care setting need to be drawn and adapted for local practice.Toward the above, field experts from infectious diseases and intensive care disciplines in Shiraz, Iran, attended a round-table discussion on 28 November 2015 to review and discuss updated epidemiologic insights on IC in ICU, the related diagnostic challenges, therapeutic approaches and proper antifungal options in ICU-admitted patients suspected for or diagnosed with IC.

\section{EPIDEMIOLOGY, INCIDENCE AND MORTALITY}

Nosocomial fungal infections comprise around 15\% of healthcare related infections among which Candida followed by Aspergillus species are found to be key culprits in invasive fungal infections (Montagna et al., 2013; Pfaller \&t Diekema, 2007). On the other hand, resistance to antifungal agents is an alarming sign for the emerging common nosocomial fungal infections (Badiee Et Alborzi, 2011). According to an elegant prospective point-prevalence investigation, Candida was regarded as the third most common cause accounting for nearly $2 \%$ of all infections (Leon et al., 2016; Timsit, Chemam, Et Bailly, 2015; Vincent et al., 2009). Moreover, a European cross-sectional survey revealed thatup to 30\% of all candidemias take place in ICUs(Marchetti et al., 2004). ICU-admitted patients are shown to be five to ten times more endangered for IC thanpatients in medical or surgical wards. Based on recent reports from North America, candida species are among the most common documented pathogens in blood cultures responsible forup to $10 \%$ of all bloodstream infections. Other studies from European ICUs have also confirmed candida to be among top tenestablished pathogens giving rise to3-5\% of bloodstream infections( Mean, et al., 2008, Chen et al., 2015; Kautzky, Staudinger, \&t Presterl, 2015) .

Based on the available evidence, IC subjects to high mortality rate ranging between 40 to 60\% (Falagas, Apostolou, \&t Pappas, 2006; Guery et al., 2009; Leroy et al., 2009), and the mortality may reach $100 \%$ under certain conditions (Kollef et al., 2012). The face of candida epidemiology has transformed over the past two decades. Though candida albicans used to be known as the dominant pathogen resulting in up to $60 \%$ of the infections, the prevalence of non-albicans species (including include C. glabrata, C. krusei, C. tropicalis and C. parapsilosis) has been on the rise lately to comprise over $50 \%$ of the infections (Arendrup, 2010; Deorukhkar \& Saini, 2016).

In an Iranian study in which 107 clinical isolates (each from one high-risk patient) were evaluated nonalbicans candida species were isolated from almost 70\% of IC cases. The most frequently isolated species was $C$. glabrata (47.7\%), followed by C. tropicalis (15\%) and C. krusei (6.5\%) (Zaini, Kordbacheh, Mahmoudi, Safara, \& Shekari, 2012). In another investigation on 855 yeast strains from different clinical specimen in Iran, over $40 \%$ of all isolated turned to be non-albicans species (Mohammadi et al., 2013). These findings are of significant clinical relevance since non-albicans candida species are generally found to be fluconazole-resistant. Taking into consideration that fluconazole is the most widely used antifungal agent against candida infection, evidence-based decision-making on choosing the proper treatment option in IC among ICU patients needs to be refocused.

In a local epidemiological study of fungal infections from Shiraz, followed by C. albicans; C. kruzei (16.1\%), C. glabrata $(13.5 \%)$, C. kefyr $(7.4 \%)$, C. parapsilosis $(4.8 \%)$, C. tropicalis $(1.7 \%)$ and other species (8.5\%) were found to collectively be responsible for over $50 \%$ of candida infections. Resistance varied based of the isolates and the corresponding antifungal agent among which the lowest MIC90 (mean inhibitory concentration-90) for non-albicans candida isolates was observed with caspofungin (0.5 $\mathrm{\mu g} / \mathrm{ml}$ )(Badiee \&t Alborzi, 2011).

\section{POTENTIAL RISK CRITERIA FOR INVASIVE FUNGAL INFECTIONS}

Colonization with various candida species which are among normal flora is not risky among healthy subjects, whereas candida species subject to dissemination 
and over growth up on extended-spectrum prolonged antibiotic therapy, burn injury, diabetes mellitus, neutropenia and immuno supression. Such a context may progressively result in disseminated candida infection/ candidemia known as IC. The condition, though not necessarily, tends to progress in cases with severe sepsis or septic shock, those who have undergone major surgeries, receiving total parenteral nutrition (TPN) and are found to have multifocal candida colonization. A fundamental clinical issue is to identify which category of patients are at increased risk for IC (Ahmadi et al., 2014; Gong et al., 2016; Hawkshead, Van Dyke, Hassig, Webber, \&t Begue, 2016; Lau et al., 2015; Liao et al., 2015; Pu et al., 2015; Rajendran et al., 2016; Sun et al., 2016).

Candida colonization may consequently turn into IC following critical illness. In the case of ICU-admitted patients, while only around 10\% of cases tend to be colonized with candida, this would be documented in up to $80 \%$ of the cases during their prolonged ICU stay, among which as many as 30\% of such patients may develop IC (Leon, Ostrosky-Zeichner, \&t Schuster, 2014). In patients who stay more than seven days in the ICU, the incidence of multifocal colonization (which is reported as an independent risk factor of IC) is dramatically increased (Kautzky et al., 2015). Research has referred to stomach $(45.6 \%)$, oropharyngeal samples (34.3\%), the trachea $(23.4 \%)$, perirectal region $(21.2 \%)$ and the urinary tract $(18.7 \%)$ as the most frequent foci for candida colonization among ICU-admitted patients. In addition, the relative risk of ICis shown to be notably increasedonce fecal $(7.5 \%$ vs. $3.2 \%, p=0.019)$ or urine samples $(9.2 \%$ vs. $5.2 \%, p=0.032)$ turn to be positive for candida. ICU-admitted patients are then suggestedto be biweekly screened (feces, urine, tracheal aspirate) to identify their potential risk for IC (Leon, Alvarez-Lerma, et al., 2009; Magill et al., 2006; Mardani et al., 2011).In the event of at least three consecutive positive samples in two or more occasions; multifocal colonization, as an independent risk of IC, needs to be considered (Gong et al., 2016; Hawkshead et al., 2016; Pittet, Monod, Suter, Frenk, \&t Auckenthaler, 1994; Rajendran et al., 2016; Sun et al., 2016).

Due to the higher rate of mortality, catheter-related candidemia needs to be differentiated from primer or intra-abdominal candidiasis-related candidemia. Catheter-related candidemia is confirmed once the same candida species are detected both in the catheter and peripheral blood samples.Such infection is known to predominantly occur through the exogenous path related tocolonization in patient's skin and the healthcare workers' hands (Leon et al., 2014).

Depending on cultures, clinical picture and the patient's risk profile, the diagnosis of IFI is generally categorized into proven, probable or possible(De Pauw et al., 2008; Moghadami et al., 2013). Proven IFI refers to positive culture result oronce histologyconfirmsthe presence of proliferous fungiin blood or other infected specimens. On the other hand, probable or possible IFIs are considered incritically-ill, neutropenic or non- neutropenic patients with extended ICU stay, multifocal colonization, sepsis or septic shock who are either positive (probable) or negative (possible) for known serum biomarkersincluding (1,3)-b-D-Glucan (BDG) or mannan antigen and anti-mannan antibody or polymerase chain reaction (PCR) (Elhoufi et al., 2014).

\section{DIAGNOSTICS MEASURES}

Though many optionsare suggested for diagnosis, none seems to be flawlesson its own. The diagnostic tools ranging from different scoring or risk-predictionmodels, to advanced laboratory measures need to be combined since IC often tend to occur with no candidemia. As such, the empirical antifungal therapy adapted based on patients' risk profile is now believed to playa key part in treating IC (Elhoufi et al., 2014; Hsu, Nguyen, Nguyen, Law, \&t Wong-Beringer, 2010; Martinez-Jimenez et al., 2016).

\section{RISK PREDICTION MODELS}

Multiple colonization, broad spectrum antibiotic therapy, total parenteral nutrition, dialysis, APACHE II (Acute Physiology and Chronic Health Evaluation II) score of $>20$ points, central venous catheters (CVC), candiduria> $105 \mathrm{cfu} / \mathrm{ml}$ and multiple transfusion are considered as some key risk factors for IC. On the other hand, some non-specific risk factors for IC include age $>65$ years, diabetes mellitus, renal failure, surgical intervention, Foley catheters, catheters inserted to vessels and long ICU stay i.e. > 7 days (Elhoufi et al., 2014).

\section{COLONIZATION INDEX}

Colonization index is known to play a prominent role in the development of IC. According to Pittet et al (Pittet et al., 1994), over one third of severely-colonized patients were found to develop documented candidiasis $(\mathrm{p}<0.01)$. The cut-off of $>0.5$ could then predictIC one weekprior tofungal cultures. Severalfuture investigation confirmed the above findings (Caggiano et al., 2011; Calandra, Roberts, Antonelli, Bassetti, \&t Vincent, 2016; Eggimann, Bille, \&t Marchetti, 2011). Based on the validation studies the positive predictive value (PPV), negative predictive value (NPV), sensitivity and specificity of the colonization index are reported 66\%, 100\%, 64\% and 69.7\%, respectively(Eggimann, Que, Revelly, \&t Pagani, 2015; 


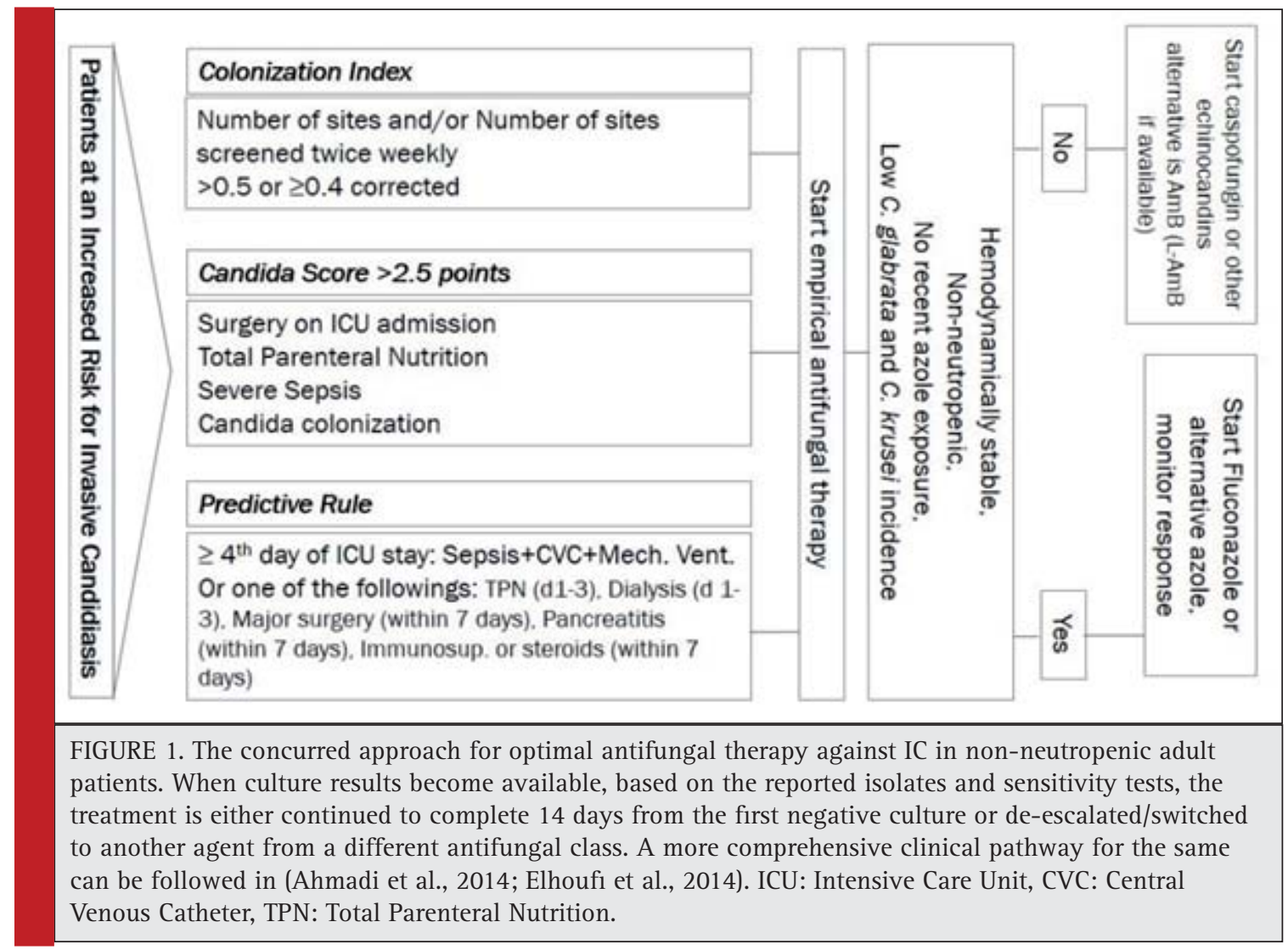

Kautzky et al., 2015; Leon et al., 2014; Posteraro et al., 2011)

The extent of colonizationcan be determined throughperiodic samples obtained from various site including, cutaneous flexure regions, nose, throat, endotracheal tube aspirate,feces and urine. Patients endangeredfor IC are advised to be biweekly screened for colonization. Colonization index is a ratio of non-sterile region samples to total number of samples. When colonization index is above 0.5 empiric antifungal therapy needs to be considered (Figure 1).

\section{CANDIDA SCORE}

Candida Score which was proposed 10 years ago by Leon et al,is in fact an upgraded form of the Colonization Index(Leon et al., 2006). This risk prediction model was validated through alarge prospective cohort in which $6 \%$ developed IC. According to the findings of Leon et al, surgery, multifocal colonization and severe sepsis acquired the odd ratios (OR) of 2.71 [95\% confidence interval (Cl): 1.45-5.06], 3.04 [95\% CI: 1.45-6.39] and 7.68 [95\% CI: 4.14-14.22] to predictIC. As such, each predictive factor received one point in candida score, except for severe sepsis which received 2 points. A Candida score of 2.5 points and more could thenpre- dict IC with a sensitivity, specificity, NPV and PPV of $81 \%, 74 \%, 98 \%$ and $16 \%$, respectively. The clinical relevance and adaptability of Candida score was validated in several subsequent reports (Leon, Ruiz-Santana, et al., 2009; Tissot et al., 2013) (Figure 1).

\section{THE OSTROSKY-ZEICHNER MODEL}

This risk-prediction model was designed to distinguishICU admitted patients who potentially require antifungal prophylaxis. The extended ICU stay was found to strongly predict the risk for ICU in a study on surgical ICU admitted patients (Paphitou, Ostrosky-Zeichner, \&t Rex, 2005). This study similarly indicated that diabetes mellitus, required acute haemodialysis, total parenteral nutrition or broad spectrum antibiotic therapy are other defining risk factors for the development of IC. IC was significantly higher in high-risk group compared to those without the aforementioned risk factors $(16.6 \%$ vs. $5.5 \%, p=0.001$ ). In fact, over three quarters of patients who went todevelop candidemia or IC were recognized by this method. Subsequent studies confirmed these results(Ahmed, et al., 2014; Yapar, 2014, Aitken et al., 2014; Lau et al., 2015; Liao et al., 2015; Gong et al., 2016;). 


\section{NON-CULTURE-BASED METHODS}

Fungal cultures become positive relatively late (Morris, Byrne, Madden, \&t Reller, 1996) and are often inadequate to diagnose deep-seated candida infections. On the other hand, performing tissue biopsies and obtaining body fluids are invasive and/or clinically impractical making them not readily accessible in routine practice (Eggimann et al., 2011). This necessitates development of other sensitive and practical diagnostic methods with high sensitivity to possibly enable timely recognition of IC. Examples of such methods include identifying cell-wall components, circulating fungal DNA, antigens and antibodies.

\section{$(1,3)-b-D-G l u c a n$}

Studies have substantiated that 1,3-b-D-Glucan (BDG) is an early biomarker of many fungal infections including candidiasis and aspergillosis (Ellis et al., 2008, Azoulay et al., 2016; Nucci et al., 2016; Posteraro et al., 2016). Based on multicenter investigations, the cut-off value of $80 \mathrm{pg} / \mathrm{ml}$ is confirmed tosuggestIC with good sensitivity and specificity except for candida parapsilosis (Pickering, Sant, Bowles, Roberts, \&t Woods, 2005). This auxiliary biomarker test reveals positive results 7-10 days prior to the established clinical diagnosis of fungal infections. Cumulative evidence has proposed BDG as an acceptable indicator of fungal infections and a reliable biomarker to start preemptive anti-fungal therapy based upon. While evidence has confirmed the correlation between BDG levels, clinical outcome and the treatment response (Takesue et al., 2004), complexity of the test and the applied cost hinder its wide availability. Some other downsides of this test include false positive results, mainly upon early days of ICU admission, and particularlyfollowing surgical interventions, immunglobulin or extended-spectrum antibiotic therapy. Though data on the kinetics of BDG are relatively scant,some reports have related the decreased BDG serum levels to therapeutic success (Ahmadi et al., 2014; Takesue et al., 2004; Tissot et al., 2013).

In case this correlation is further confirmed by clinical investigations, BDG may possibly be considered as a tool in assessing response to antifungal therapy. While the test is shown to trace BDG levels even in other body fluids including cerebro-spinal and peritonealfluids as well as bronchoalveolar secretions (Lyons et al., 2015; Mutschlechner et al., 2015), it needs to be validated for extended clinical use. Lately, the European Society of Clinical Microbiology and Infectious Diseases (ESCMD), the Society of Critical Care Medicine (SCCM), and the European Society of Intensive Care Medicine (ESICM), have included BDG testing in their recommendations based on the existing level-II evidence (Elhoufi et al., 2014; Hope et al., 2012; Ullmann et al., 2012).

\section{Mannan antigen and anti-mannan antibody}

In case of invasive candidiasis, mannan, as a component of candida cell wall, circulates in the bloodstream. In practice, a range of immunoassay-based and latex agglutination methods are usedto detect mannan (Schuetz, 2013). The combined detection of mannan antigen and anti-mannan antibody is known to yield a better sensitivity. Based on a recent meta-analysis, the sensitivity and specificity ofmannan antigen and anti-mannan antibody tests were $(58 \%, 93 \%)$ and $(59 \%, 83 \%)$, respectively. Furthermore, the sensitivity and specificity of the combined test is shown to be improved (83\% and $86 \%$, respectively) for C. albicans, C. glabrata and C. tropicalis infections, when these investigations were combined (Mikulska et al., 2010). Despite the existing body of evidence, further investigations (examining homogeneous patient groups with IFIs) are deemed necessary to substantiate the positive and negative predictive values of the test and define the its role in routine practice.

\section{Detection of Candida nucleic acids by PCR}

Despite the potentially informative nature of fungal DNA detection in the practice of clinical mycology, the DNA disengagement secondary to human cell-lysis as well as the contamination from other saprophytic or pathogen fungi may lead to its false positive results. This makes the test challenging. Nevertheless, studies have demonstrated that PCR is appropriate for timely detection of candidemia and the detection of organic fragments of the multicopy gene. The test is also shown to detect non-viable organisms quicker than the culture where different platforms and target genes (other than blood samples) are investigated during the test (Avni, Leibovici, \&t Paul, 2011).

A recent meta-analysis has reported favorable overall sensitivity and specificity (95\% and 92\%) of PCR in detecting IC. This elegant report included 54 studies and 4894 patients among which 963 had proven, probable or possible IC (Avni et al., 2011).Despite the above, direct molecular detection of candida is not yet considered as a standard method and until validation for routine clinical use, the position of pan-fungal PCR test or further molecular methods in early detection of ICremainsindefinite.

\section{CULTURE-BASED DIAGNOSTICS}

The culture-base diagnosis of IFIs are recognized as the gold standard(Ahmadi et al., 2014). Based on the evidence, the sensitivity of blood cultures for invasive candisiasisranges between 50-70\%. This may even be decreased in neutropenic patients andthose receiving antifungal therapy (Ahmadi et al., 2014; Ullmann et al., 2012). When catheter-related infections are suspected, 
to establish a source control, samples should also be obtained from the catheters(Ullmann et al., 2012).

When culture results turn positive for candida species, in addition to performing resistance tests, it is important to note that minimal inhibitory concentration (MIC) values may also affect the therapy. The documentation of the candida species is time-consuming may require several days following positive results. Meanwhile, this may be fast-tracked through some novel techniques including PNA-FISH (Peptide Nucleic Acid Fluorescence In Situ Hybridization) and MALDI-TOF MS (Matrix Assisted Laser Desorption Ionization Time-of-Flight Mass Spectrometry) which are not necessarily available and/or validated in everyday practice (Heil et al., 2012; Saracli, Fothergill, Sutton, \& Wiederhold, 2015).

Despite the fact that the definite diagnosis of IFI relies on blood cultures, they certainly cannot be classified among the early diagnostic strategies. Culture results are typically available after several days while as-earlyas-possible clinical decision is often necessary to save patient's life. Based on the above, cultures may largely be used as confirmatory tests to continue an already commenced empirical antifungal regimen to de-escalate it to other options (Elhoufi et al., 2014).

\section{THERAPEUTIC APPROACHES TOWARDS INVASIVE CANDIDIASIS}

The clinical outcome in patients with invasive candidiasis largely depends on the timeliness in antifungal therapy (Hope et al., 2012; Ullmann et al., 2012). Many clinical investigations have clearly shown that delayed approach in antifungal therapy has a negative impact on survival (Ahmadi et al., 2014; Ahmed et al., 2014; Blot, Vandewoude, Hoste, \&t Colardyn, 2002; Corona, Cislaghi, \&t Singer, 2008; Elhoufi et al., 2014; Garey et al., 2006; Mardani et al., 2011; Morrell, Fraser, \&t Kollef, 2005; Skrobik \&t Laverdiere, 2013; Viaggi, Tascini, \&t Menichetti, 2014; Yildirmak, Gedik, Simsek, Iris, \&t Gucuyener, 2013). Based upon the diagnostic possibility of IFIs, various strategies including prophylaxis, empirical, preemptive, or targeted therapy may be pursued.

\section{PROPHYLAXIS}

Prophylactic antifungal therapy is used to prevent IFIs, namely invasive candida infection in patients who are asymptomatic yet at high-risk (Moghadami et al., 2013). Though fluconazole is the mostly used prophylactic option in general, echinocandins have also been successfully used (Senn et al., 2009). Unlike the significant position of prophylaxis in immunocompromised hosts (Fortun et al., 2016; Mardani et al., 2011; Moghadami et al., 2013), this approach is not commonly recommended in non-neutropenic ICU-admitted patients (Ullmann et al., 2012).

\section{EMPIRICAL THERAPY}

As defined by ESCMID (Arendrup et al., 2014; Ullmann et al., 2012), empirical approach is warranted in patients withpersistent fever despite adequate antibiotic therapy who are found to be at high risk forinvasive candidiasis based on their increased risk scores.

\section{PRE-EMPTIVE THERAPY}

In case of microbiological evidence of IFIs (1-3 BDG biomarker, mannan/anti-mannan double test or detection of fungal nucleic acid by PCR) in clinically-suspected cases, pre-emptive approach become warranted as defined by ESCMID (Ullmann et al., 2012). Such IFIs are typically categorized into "possible" or "probable".

\section{TARGETED THERAPY}

The antifungal therapy can be adapted to achieve favorable results as targeted therapy, once the sensitivity of anti-candidaoptions as well as their MIC from the blood culture or other specimen is identified. Though this is considered as gold-standard, availability of cultures results is not timely (Badiee \&t Alborzi, 2011; Eggimann et al., 2011; Mean et al., 2008; Ullmann et al., 2012).

\section{CHOICE OF THE ANTIFUNGAL AGENT AND LENGTH OF THERAPY}

A number of international guidelines and national consensus statements (from the IFI-CF forums across Iran) are available to optimize our practice upon choosing the appropriate agents in IFI management in criticallyill patients (Ahmadi et al., 2014; Elhoufi et al., 2014; Kontoyiannis, 2001; Mardani et al., 2011; Moghadami et al., 2013; Pappas et al., 2016; Patterson et al., 2016; Ullmann et al., 2012). Meanwhile, local epidemiology and resistance profiles need to be established and taken into account when considering an antifungal option in a given clinical scenario.

Considering the available guidelines, local epidemiology (Badiee Et Alborzi, 2011; Mohammadi et al., 2013; Zaini et al., 2012), and expert panels' inputs from the IFI-CF, Shiraz, the concurred approachto antifungal therapy against IC in non-neutropenic adult patients is summarized in Figure 1.

With regard to the initiation and duration of therapy, in case of a positive blood culture for candida, empiric antifungal therapy should immediately be commenced followed by daily blood cultures. The patient needs to be treated for a minimum of 14 days following the first 
negative culture. If candidemia is present, fundoscopic examination becomes crucial to exclude intraocular candidiasis (Pappas et al., 2016; Ullmann et al., 2012).

\section{SUMMARY AND CONCLUSIVE REMARKS/ RECOMMENDATIONS FROM THE IFI-CF, SHIRAZ}

The burden of IFIs needs to receive a more focused clinical attentionespecially in our high-risk, critically-ill, ICU-admitted patients. Despite the recent progress indiagnosis and treatment of IFIs, this clinically-significant issue continues to often be overlooked. Timely diagnosis and properantifungal therapy is the only chance for improved survival in critically-ill patients with IFIs. As far as concernedby the practice guidelines, empirical and pre-emptive approaches tend to the most promising strategies (Ahmadi et al., 2014; Elhoufı et al., 2014; He et al., 2015; Liu et al., 2015; Pappas et al., 2016; Patterson et al., 2016; Shi et al., 2015; Ullmann et al., 2012). Validation and inclusion of fungalbiomarker assays and DNA tests in our practicewould potentially improve diagnostic accuracy and enable earlier treatment approaches. Nonetheless, several concerns need to be resolved not only on availability and validity of such tests in routine practice, but also the remaining key issues in diagnostics and therapy as well as determination of the treatment duration.

The present IFI-CF (Invasive Fungal Infections-Clinical Forum) brought together an interdisciplinary panel of experts from infectious diseases and intensive care fields in Shiraz to view the available evidence, taking into consideration recent practice guidelines on IFIs management in the ICU. The panelaimed to arrive at an agreed position in current clinical practice of IFIs in ICU. The following remarks emerged from the IFI-CF's discussions on epidemiology, the range of pathophysiology from colonization to the invasive infections, risk prediction, diagnosis and treatment perspectives:

1. The necessity of extending local epidemiology studies on candida isolates and antifungals' sensitivity in patients admitted to ICUs

2. Identifying the impact of adherence to the current local consensus as well as other local and international practice guidelines on the clinical outcome of ICU-admitted patients at risk for IFIs

3. Considering the use of risk prediction models, namely the 'Candida Score', to identify critically-ill patients eligible for the empirical antifungal approach

4. Designing clinical studies in the ICU to consider invasive infections rather than colonization and to identify the role of fungal biomarkers (1-3 BDG biomarker, mannan/anti-mannan double test or detection of fungal nucleic acid by PCR) in optimizing our clinical approaches.

5. Capitalizing on a 'working-team concept', with the medical microbiologists involved, to further address key questions including 'when to start treatment?', 'what strategy to pursue?'and 'which option to take?' in IFIs among ICU-admitted patients.

\section{APPENDIX}

The IFI-CF received contribution from the following collaborators and consultants from Shiraz University of Medical Sciences, Shiraz, Iran (sorted alphabetically):

Asadpour, Elham (Pharmacology); Badiee, Parisa (Medical Mycology); Dabiri, Gholamreza (Intensive Care); Fallahi, Mohammad-Javad (Pulmonology); Ghayumi, Seyed Mohammad-Ali (Pulmonology); HaddadBakhodaei, Hosein (Intensive Care); Haghbin, Saeedeh (Pediatrics, Intensive Care); Khaloo, Vahid (Intensive Care); Mackie, Mandana (Intensive Care); Momeni, Behrooz (Pulmonology); Mortazavi, Shahram (Infectious Diseases); Pouladfar, Gholamreza (Pediatrics, Infectious Diseases); Sabetian, Golnar (Intensive Care); Savaie, Mohsen (Intensive Care); Vazin, Afsaneh (Clinical Pharmacy); Zomorodian, Kamiar (Medical Mycology).

\section{ACKNOWLEDGMENTS}

Authors would like to thank Dr. Dindoust P, Salarian A, Hejazi-Farahmand SAR, for supporting this clinical forum. The 'IFI-CF, Shiraz' received scientific and administrative support from Shiraz Anesthesiology and Critical Care Research Center, Shiraz University of Medical Sciences, Shiraz, Iran, as well as the MSD Medical team at Behstan Darou PJS, Tehran, Iran.

\section{COMPETING INTEREST}

The present report outlined the communications andexperts' opinions during the IFI-CF held on 28 November 2015, Shiraz, Iran. The authors declare no competing interest upondata review, talk delivery during the meeting, interactivediscussions and preparation of the present report. MN provided medical consultancy to Behestan Medical Scientific Committee, Behestan Group, Tehran, Iran.

\section{REFERENCES}

Ahmadi, A., Ardehali, S. H., Beigmohammadi, M. T., Hajiabdolbaghi, M., Hashemian, S. M., Kouchek, M. TorabiNami, M. (2014). Invasive candidiasis in intensive care unit; consensus statement from an Iranian panel of experts, 
July 2013. JRSM Open, 5(3), 2042533313517689. doi: $10.1177 / 2042533313517689$

Ahmed, A., Azim, A., Baronia, A. K., Marak, K. R., \&t Gurjar, M. (2014). Risk prediction for invasive candidiasis. Indian J Crit Care Med, 18(10), 682-688. doi: 10.4103/0972-5229.142178

Aitken, S. L., Beyda, N. D., Shah, D. N., Palmer, H. R., Lasco, T. M., Koo, H., \& Garey, K. W. (2014). Clinical practice patterns in hospitalized patients at risk for invasive candidiasis: role of antifungal stewardship programs in an era of rapid diagnostics. Ann Pharmacother, 48(6), 683-690. doi: $10.1177 / 1060028014529928$

Arendrup, M. C. (2010). Epidemiology of invasive candidiasis. Curr Opin Crit Care, 16(5), 445-452. doi: 10.1097/ MCC.0b013e32833e84d2

Arendrup, M. C., Boekhout, T., Akova, M., Meis, J. F., Cornely, 0. A., Lortholary, O. et al., European Confederation of Medical, M. (2014). ESCMID and ECMM joint clinical guidelines for the diagnosis and management of rare invasive yeast infections. Clin Microbiol Infect, 20 Suppl 3, 76-98. doi: 10.1111/14690691.12360

Avcu, G., Karapinar, D. Y., Yazici, P., Duyu, M., Polat, S. H., Atabay, B. et al., Karapinar, B. (2016). Difficult diagnosis of invasive fungal infection predominantly involving the lower gastrointestinal tract in acute lymphoblastic leukaemia. Med Mycol Case Rep, 11, 1-4. doi: 10.1016/j.mmcr.2016.01.005

Avni, T., Leibovici, L., \& Paul, M. (2011). PCR diagnosis of invasive candidiasis: systematic review and meta-analysis. J Clin Microbiol, 49(2), 665-670. doi: 10.1128/JCM.01602-10

Azoulay, E., Guigue, N., Darmon, M., Mokart, D., Lemiale, V., Kouatchet, A., Pene, F. (2016). (1, 3)-beta-D-glucan assay for diagnosing invasive fungal infections in critically ill patients with hematological malignancies. Oncotarget. doi: 10.18632/ oncotarget.7471

Badiee, P., \& Alborzi, A. (2011). Susceptibility of clinical Candida species isolates to antifungal agents by E-test, Southern Iran: A five year study. Iran J Microbiol, 3(4), 183-188.

Blot, S. I., Vandewoude, K. H., Hoste, E. A., \&t Colardyn, F. A. (2002). Effects of nosocomial candidemia on outcomes of critically ill patients. Am J Med, 113(6), 480-485.

Caggiano, G., Puntillo, F., Coretti, C., Giglio, M., Alicino, I., Manca, F., Montagna, M. T. (2011). Candida colonization index in patients admitted to an ICU. Int J Mol Sci, 12(10), 70387047. doi: 10.3390/ijms12107038

Calandra, T., Roberts, J. A., Antonelli, M., Bassetti, M., \&t Vincent, J. L. (2016). Diagnosis and management of invasive candidiasis in the ICU: an updated approach to an old enemy. Crit Care, 20(1), 125. doi: 10.1186/s13054-016-1313-6

Chen, J., Yu, X., Zhou, Y., Zhang, Y., Zhu, J., Xie, L . He, Z. (2015). Integrated measures for prevention of invasive Candida infections in preterm infants in a Chinese neonatal intensive care unit. Am J Infect Control, 43(12), 1321-1325. doi: 10.1016/j.ajic.2015.07.011

Corona, A., Cislaghi, F. \&t Singer, M. (2008). Timely selection of adequate antifungal therapy for candidemia in the critically ill: don't let the yeast rise! Crit Care Med, 36(11), 3097-3098. doi: 10.1097/CCM.0b013e31818b9375

De Pauw, B., Walsh, T. J., Donnelly, J. P., Stevens, D. A., Edwards, J. E., Calandra, T., et al., Infectious Diseases Mycoses Study Group Consensus, G. (2008). Revised definitions of invasive fungal disease from the European Organization for Research and Treatment of Cancer/Invasive Fungal Infections Cooperative Group and the National Institute of Allergy and Infectious Diseases Mycoses Study Group (EORTC/MSG) Consensus Group. Clin Infect Dis, 46(12), 1813-1821. doi: 10.1086/588660

Deorukhkar, S. C. \& Saini S. (2016). Echinocandin Susceptibility Profile of Fluconazole Resistant Candida Species Isolated from Blood Stream Infections. Infect Disord Drug Targets, 16(1), 63-68.

Eggimann, P., Bille, J., \& Marchetti, 0. (2011). Diagnosis of invasive candidiasis in the ICU. Ann Intensive Care, 1, 37. doi: 10.1186/2110-5820-1-37

Eggimann, P., Que, Y. A., Revelly, J. P., \&t Pagani, J. L. (2015). Preventing invasive candida infections. Where could we do better? J Hosp Infect, 89(4), 302-308. doi: 10.1016/j. jhin.2014.11.006

Elhoufi, A., Ahmadi, A., Asnaashari, A. M., Davarpanah, M. A., Bidgoli, B. F., Moghaddam, 0. M. et al., Zand, F. (2014). Invasive candidiasis in critical care setting, updated recommendations from "Invasive Fungal Infections-Clinical Forum", Iran. World J Crit Care Med, 3(4), 102-112. doi: 10.5492/wjccm. v3.i4.102

Ellis, M., Al-Ramadi, B., Finkelman, M., Hedstrom, U., Kristensen, J., Ali-Zadeh, H., \& Klingspor, L. (2008). Assessment of the clinical utility of serial beta-D-glucan concentrations in patients with persistent neutropenic fever. J Med Microbiol, 57(Pt 3), 287-295. doi: 10.1099/jmm.0.47479-0

Falagas, M. E., Apostolou, K. E., \&t Pappas, V. D. (2006). Attributable mortality of candidemia: a systematic review of matched cohort and case-control studies. Eur J Clin Microbiol Infect Dis, 25(7), 419-425. doi: 10.1007/s10096-006-0159-2

Fortun, J., Muriel, A., Martin-Davila, P., Montejo, M., Len, 0., Torre-Cisneros, J. et al., Red Espanola de Investigacion en Patologia, I. (2016). Caspofungin versus fluconazole as prophylaxis of invasive fungal infection in high-risk liver transplantation recipients: A propensity score analysis. Liver Transpl, 22(4), 427-435. doi: 10.1002/lt.24391

Garey, K. W., Rege, M., Pai, M. P., Mingo, D. E., Suda, K. J., Turpin, R. S., \&t Bearden, D. T. (2006). Time to initiation of fluconazole therapy impacts mortality in patients with candidemia: a multi-institutional study. Clin Infect Dis, 43(1), 25-31. doi: 10.1086/504810

Gong, X., Luan, T., Wu, X., Li, G., Qiu, H., Kang, Y., Zang, B. (2016). Invasive candidiasis in intensive care units in China: Risk factors and prognoses of Candida albicans and non-albicans Candida infections. Am J Infect Control, 44(5), e59-63. doi: 10.1016/j.ajic.2015.11.028

Guery, B. P., Arendrup, M. C., Auzinger, G., Azoulay, E., Borges Sa, M., Johnson, E. M. et al., Kullberg, B. J. (2009). Management of invasive candidiasis and candidemia in adult non- 
neutropenic intensive care unit patients: Part I. Epidemiology and diagnosis. Intensive Care Med, 35(1), 55-62. doi: 10.1007/ s00134-008-1338-7

Hawkshead, J. J., 3rd, Van Dyke, R. B., Hassig, S. E., Webber, L. S., Ct Begue, R. E. (2016). Species-based comparison of disease severity and risk factors for disseminated Candida infections in pediatric patients. Infect Drug Resist, 9, 59-70. doi: 10.2147/ IDR.S102490

He, S., Hang, J. P., Zhang, L., Wang, F., Zhang, D. C., \&t Gong, F. H. (2015). A systematic review and meta-analysis of diagnostic accuracy of serum 1,3-beta-D-glucan for invasive fungal infection: Focus on cutoff levels. J Microbiol Immunol Infect, 48(4), 351-361. doi: 10.1016/j.jmii.2014.06.009

Heil, E. L., Daniels, L. M., Long, D. M., Rodino, K. G., Weber, D. J., \& Miller, M. B. (2012). Impact of a rapid peptide nucleic acid fluorescence in situ hybridization assay on treatment of Candida infections. Am J Health Syst Pharm, 69(21), 1910-1914. doi: 10.2146/ajhp 110604

Hope, W. W., Castagnola, E., Groll, A. H., Roilides, E., Akova, M., Arendrup, M. C., Group, E. F. I. S. (2012). ESCMID* guideline for the diagnosis and management of Candida diseases 2012: prevention and management of invasive infections in neonates and children caused by Candida spp. Clin Microbiol Infect, 18 Suppl 7, 38-52. doi: 10.1111/1469-0691.12040

Hsu, D. I., Nguyen, M., Nguyen, L., Law, A., \& Wong-Beringer, A. (2010). A multicentre study to evaluate the impact of timing of caspofungin administration on outcomes of invasive candidiasis in non-immunocompromised adult patients. J Antimicrob Chemother, 65(8), 1765-1770. doi: 10.1093/jac/dkq216

Kautzky, S., Staudinger, T., \&t Presterl, E. (2015). Invasive Candida infections in patients of a medical intensive care unit: attempt of improving diagnosis by quantifying the colonization. Wien Klin Wochenschr, 127(3-4), 132-142. doi: 10.1007/ s00508-014-0644-Z

Kollef, M., Micek, S., Hampton, N., Doherty, J. A., \&t Kumar, A. (2012). Septic shock attributed to Candida infection: importance of empiric therapy and source control. Clin Infect Dis, 54(12), 1739-1746. doi: 10.1093/cid/cis305

Kontoyiannis, D. P. (2001). A clinical perspective for the management of invasive fungal infections: focus on IDSA guidelines. Infectious Diseases Society of America. Pharmacotherapy, 21(8 Pt 2), 175S-187S.

Lau, A. F., Kabir, M., Chen, S. C., Playford, E. G., Marriott, D. J., Jones, M.,Sorrell, T. C. (2015). Candida colonization as a risk marker for invasive candidiasis in mixed medical-surgical intensive care units: development and evaluation of a simple, standard protocol. J Clin Microbiol, 53(4), 1324-1330. doi: 10.1128/JCM.03239-14

Leon, C., Alvarez-Lerma, F., Ruiz-Santana, S., Leon, M. A., Nolla, J., Jorda, R., Group, E. S. (2009). Fungal colonization and/or infection in non-neutropenic critically ill patients: results of the EPCAN observational study. Eur J Clin Microbiol Infect Dis, 28(3), 233-242. doi: 10.1007/s 10096-008-0618-Z

Leon, C., Ostrosky-Zeichner, L., \& Schuster, M. (2014). What's new in the clinical and diagnostic management of invasive candidiasis in critically ill patients. Intensive Care Med, 40(6), 808-819. doi: 10.1007/s00134-014-3281-0

Leon, C., Ruiz-Santana, S., Saavedra, P., Almirante, B., NollaSalas, J., Alvarez-Lerma, F., Group, E. S. (2006). A bedside scoring system ("Candida score") for early antifungal treatment in nonneutropenic critically ill patients with Candida colonization. Crit Care Med, 34(3), 730-737. doi: 10.1097/01. CCM.0000202208.37364.7D

Leon, C., Ruiz-Santana, S., Saavedra, P., Castro, C., Loza, A., Zakariya, I., Cava Trem Study, G. (2016). Contribution of Candida biomarkers and DNA detection for the diagnosis of invasive candidiasis in ICU patients with severe abdominal conditions. Crit Care, 20(1), 149. doi: 10.1186/s13054-0161324-3

Leon, C., Ruiz-Santana, S., Saavedra, P., Galvan, B., Blanco, A., Castro, C., Cava Study, G. (2009). Usefulness of the "Candida score" for discriminating between Candida colonization and invasive candidiasis in non-neutropenic critically ill patients: a prospective multicenter study. Crit Care Med, 37(5), 16241633. doi: 10.1097/CCM.0b013e31819daa14

Lepak, A., \& Andes, D. (2011). Fungal sepsis: optimizing antifungal therapy in the critical care setting. Crit Care Clin, 27(1), 123-147. doi: 10.1016/j.ccc.2010.11.001

Leroy, O., Gangneux, J. P., Montravers, P., Mira, J. P., Gouin, F., Sollet, J. P. AmarCand Study, G. (2009). Epidemiology, management, and risk factors for death of invasive Candida infections in critical care: a multicenter, prospective, observational study in France (2005-2006). Crit Care Med, 37(5), 1612-1618. doi: 10.1097/CCM.0b013e31819efac0

Liao, X., Qiu, H., Li, R., Guo, F., Liu, W., Kang, M., China, S. T. (2015). Risk factors for fluconazole-resistant invasive candidiasis in intensive care unit patients: An analysis from the China Survey of Candidiasis study. J Crit Care, 30(4), 862 e861-865. doi: 10.1016/j.jcrc.2015.04.002

Liu, Y. C., Chien, S. H., Fan, N. W., Hu, M. H., Gau, J. P., Liu, C. J., Tzeng, C. H. (2015). Incidence and risk factors of probable and proven invasive fungal infection in adult patients receiving allogeneic hematopoietic stem cell transplantation. J Microbiol Immunol Infect. doi: 10.1016/j.jmii.2015.01.002

Lyons, J. L., Thakur, K. T., Lee, R., Watkins, T., Pardo, C. A., Carson, K. A., Zhang, S. X. (2015). Utility of measuring (1,3)-beta$\mathrm{d}$-glucan in cerebrospinal fluid for diagnosis of fungal central nervous system infection. J Clin Microbiol, 53(1), 319-322. doi: 10.1128/JCM.02301-14

Magill, S. S., Swoboda, S. M., Johnson, E. A., Merz, W. G., Pelz, R. K., Lipsett, P. A., \&t Hendrix, C. W. (2006). The association between anatomic site of Candida colonization, invasive candidiasis, and mortality in critically ill surgical patients. Diagn Microbiol Infect Dis, 55(4), 293-301. doi: 10.1016/j.diagmicrobio.2006.03.013

Marchetti, O., Bille, J., Fluckiger, U., Eggimann, P., Ruef, C., Garbino, J. Fungal Infection Network of, S. (2004). Epidemiology of candidemia in Swiss tertiary care hospitals: secular trends, 1991-2000. Clin Infect Dis, 38(3), 311-320. doi: $10.1086 / 380637$ 
Mardani, M., Tabarsi, P., Yadegarinia, D., Talebi Taher, M., Najafi, N., Hajabdolbaghi, M. et al., Kamali, M. (2011). Treatment of Invasive Fungal Infection: Recommendations from Scientific Leaders' Meeting on November 3rd, 2011 Tehran Iran. Archives of Clinical Infectious Disease, 6(4), 178-181.

Martinez-Jimenez, M. C., Munoz, P., Valerio, M., Vena, A., Guinea, J. \&t Bouza, E. (2016). Combination of Candida biomarkers in patients receiving empirical antifungal therapy in a Spanish tertiary hospital: a potential role in reducing the duration of treatment. J Antimicrob Chemother. doi: 10.1093/ jac/dkw193

Mean, M., Marchetti, O., \&t Calandra, T. (2008). Bench-to-bedside review: Candida infections in the intensive care unit. Crit Care, 12(1), 204. doi: 10.1186/cc6212

Mikulska, M., Calandra, T., Sanguinetti, M., Poulain, D., Viscoli, C., \& Third European Conference on Infections in Leukemia, G. (2010). The use of mannan antigen and anti-mannan antibodies in the diagnosis of invasive candidiasis: recommendations from the Third European Conference on Infections in Leukemia. Crit Care, 14(6), R222. doi: 10.1186/cc9365

Moghadami, M., Ramzi, M., Dehghani, M., Davarpanah, M., Nourani Khojasteh, H., Vojdani, R. et al., Torabi-Nami, M. (2013). Invasive Fungal Infection Prophylaxis in Immunocompromised Hosts; the Consensus from Shiraz IFI Study Group, December 2012, Shiraz, Iran International Journal of Internal Medicine, 2(2). doi: 10.5923/j.ijim.20130202.02

Mohammadi, R., Mirhendi, H., Rezaei-Matehkolaei, A., Ghahri, M., Shidfar, M. R., Jalalizand, N., \&t Makimura, K. (2013). Molecular identification and distribution profile of Candida species isolated from Iranian patients. Med Mycol, 51(6), 657663. doi: 10.3109/13693786.2013.770603

Montagna, M. T., Caggiano, G., Lovero, G., De Giglio, 0., Coretti, C., Cuna, T. Puntillo, F. (2013). Epidemiology of invasive fungal infections in the intensive care unit: results of a multicenter Italian survey (AURORA Project). Infection, 41(3), 645-653. doi: 10.1007/s15010-013-0432-0

Morrell, M., Fraser, V. J., \&t Kollef, M. H. (2005). Delaying the empiric treatment of candida bloodstream infection until positive blood culture results are obtained: a potential risk factor for hospital mortality. Antimicrob Agents Chemother, 49(9), 3640-3645. doi: 10.1128/AAC.49.9.3640-3645. 2005

Morris, A. J., Byrne, T. C., Madden, J. F., \&t Reller, L. B. (1996). Duration of incubation of fungal cultures. J Clin Microbiol, 34(6), 1583-1585.

Mutschlechner, W., Risslegger, B., Willinger, B., Hoenigl, M., Bucher, B., Eschertzhuber, S., \& Lass-Florl, C. (2015). Bronchoalveolar Lavage Fluid (1,3)beta-D-Glucan for the Diagnosis of Invasive Fungal Infections in Solid Organ Transplantation: A Prospective Multicenter Study. Transplantation, 99(9), e140144. doi: 10.1097/TP.0000000000000635

Nucci, M., Nouer, S. A., Esteves, P., Guimaraes, T., Breda, G., Miranda, B. G. Colombo, A. L. (2016). Discontinuation of empirical antifungal therapy in ICU patients using 1,3-betad-glucan. J Antimicrob Chemother. doi: 10.1093/jac/dkw 188
Paphitou, N. I., Ostrosky-Zeichner, L., \& Rex, J. H. (2005). Rules for identifying patients at increased risk for candidal infections in the surgical intensive care unit: approach to developing practical criteria for systematic use in antifungal prophylaxis trials. Med Mycol, 43(3), 235-243.

Pappas, P. G., Kauffman, C. A., Andes, D. R., Clancy, C. J., Marr, K. A., Ostrosky-Zeichner, L. et al., Sobel, J. D. (2016). Clinical Practice Guideline for the Management of Candidiasis: 2016 Update by the Infectious Diseases Society of America. Clin Infect Dis, 62(4), e1-50. doi: 10.1093/cid/civ933

Patterson, T. F., Thompson, G. R., 3rd, Denning, D. W., Fishman, J. A., Hadley, S., Herbrecht, R. et al., Bennett, J. E. (2016). Practice Guidelines for the Diagnosis and Management of Aspergillosis: 2016 Update by the Infectious Diseases Society of America. Clin Infect Dis. doi: 10.1093/cid/ciw326

Pfaller, M. A., \&t Diekema, D. J. (2007). Epidemiology of invasive candidiasis: a persistent public health problem. Clin Microbiol Rev, 20(1), 133-163. doi: 10.1128/CMR.00029-06

Pickering, J. W., Sant, H. W., Bowles, C. A., Roberts, W. L., Et Woods, G. L. (2005). Evaluation of a (1->3)-beta-D-glucan assay for diagnosis of invasive fungal infections. J Clin Microbiol, 43(12), 5957-5962. doi: 10.1128/JCM.43.12.59575962.2005

Pittet, D., Monod, M., Suter, P. M., Frenk, E., \& Auckenthaler, R. (1994). Candida colonization and subsequent infections in critically ill surgical patients. Ann Surg, 220(6), 751-758.

Posteraro, B., De Pascale, G., Tumbarello, M., Torelli, R., Pennisi, M. A., Bello, G. Antonelli, M. (2011). Early diagnosis of candidemia in intensive care unit patients with sepsis: a prospective comparison of (1-->3)-beta-D-glucan assay, Candida score, and colonization index. Crit Care, 15(5), R249. doi: 10.1186/cc10507

Posteraro, B., Tumbarello, M., De Pascale, G., Liberto, E., Vallecoccia, M. S., De Carolis, E. Antonelli, M. (2016). (1,3)-betad-Glucan-based antifungal treatment in critically ill adults at high risk of candidaemia: an observational study. J Antimicrob Chemother. doi: 10.1093/jac/dkw112

Pu, S., Niu, S., Zhang, C., Xu, X., Qin, M., Huang, S., \&t Zhang, L. (2015). Epidemiology, antifungal susceptibilities, and risk factors for invasive candidiasis from 2011 to 2013 in a teaching hospital in southwest China. J Microbiol Immunol Infect. doi: 10.1016/j.jmii.2015.01.005

Rajendran, R., Sherry, L., Nile, C. J., Sherriff, A., Johnson, E. M., Hanson, M. F. et al., Ramage, G. (2016). Biofilm formation is a risk factor for mortality in patients with Candida albicans bloodstream infection-Scotland, 2012-2013. Clin Microbiol Infect, 22(1), 87-93. doi: 10.1016/j.cmi.2015.09.018

Saracli, M. A., Fothergill, A. W., Sutton, D. A., \&t Wiederhold, N. P. (2015). Detection of triazole resistance among Candida species by matrix-assisted laser desorption/ionization-time of flight mass spectrometry (MALDI-TOF MS). Med Mycol, 53(7), 736-742. doi: 10.1093/mmy/myv046

Schuetz, A. N. (2013). Invasive fungal infections: biomarkers and molecular approaches to diagnosis. Clin Lab Med, 33(3), 505-525. doi: 10.1016/j.cll.2013.03.009 
Senn, L., Eggimann, P., Ksontini, R., Pascual, A., Demartines, N., Bille, J.Marchetti, 0. (2009). Caspofungin for prevention of intra-abdominal candidiasis in high-risk surgical patients. Intensive Care Med, 35(5), 903-908. doi: 10.1007/s00134-0091405-8

Shi, J. M., Pei, X. Y., Luo, Y., Tan, Y. M., Tie, R. X., He, J. S. Huang, H. (2015). Invasive fungal infection in allogeneic hematopoietic stem cell transplant recipients: single center experiences of 12 years. J Zhejiang Univ Sci B, 16(9), 796-804. doi: 10.1631/jzus.B1500005

Skrobik, Y., \&t Laverdiere, M. (2013). Why Candida sepsis should matter to ICU physicians. Crit Care Clin, 29(4), 853-864. doi: 10.1016/j.ccc.2013.06.007

Solmaz, S., Korur, A., Yeral, M., Gereklioglu, C., Ulusan, S. N., Boga, C., \& Ozdogu, H. (2016). Active Invasive Fungal Infection in a Patient With Severe Aplastic Anemia. Exp Clin Transplant. doi: 10.6002/ect.2015.0307

Sun, H., Chen, Y., Zou, X., Li, H., Yin, X., Qin, H.Han, L. (2016). Occurrence of oral Candida colonization and its risk factors among patients with malignancies in China. Clin Oral Investig, 20(3), 459-467. doi: 10.1007/s00784-015-1524-2

Takesue, Y., Kakehashi, M., Ohge, H., Imamura, Y., Murakami, Y., Sasaki, M., Sueda, T. (2004). Combined assessment of beta-D-glucan and degree of candida colonization before starting empiric therapy for candidiasis in surgical patients. World J Surg, 28(6), 625-630. doi: 10.1007/s00268-0047302-y

Timsit, J. F., Chemam, S., \& Bailly, S. (2015). Empiric/pre-emptive anti-Candida therapy in non-neutropenic ICU patients. F1000Prime Rep, 7, 21. doi: 10.12703/P7-21

Tissot, F., Lamoth, F., Hauser, P. M., Orasch, C., Fluckiger, U., Siegemund, M.Fungal Infection Network of, S. (2013). beta-glucan antigenemia anticipates diagnosis of blood culture-negative intraabdominal candidiasis. Am J Respir
Crit Care Med, 188(9), 1100-1109. doi: 10.1164/rccm.20121120690C

Ullmann, A. J., Cornely, 0. A., Donnelly, J. P., Akova, M., Arendrup, M. C., Arikan-Akdagli, S., Group, E. F. I. S. (2012). ESCMID* guideline for the diagnosis and management of Candida diseases 2012: developing European guidelines in clinical microbiology and infectious diseases. Clin Microbiol Infect, 18 Suppl 7, 1-8. doi: 10.1111/1469-0691.12037

Viaggi, B., Tascini, C., \& Menichetti, F. (2014). The definition of starting point for evaluation of timely adequate antifungal therapy. Intensive Care Med, 40(12), 1973. doi: 10.1007/ s00134-014-3426-1

Vincent, J. L., Rello, J., Marshall, J., Silva, E., Anzueto, A., Martin, C. D. Investigators, E. I. G. o. (2009). International study of the prevalence and outcomes of infection in intensive care units. JAMA, 302(21), 2323-2329. doi: 10.1001/ jama.2009.1754

Wu, G. X., Khojabekyan, M., Wang, J., Tegtmeier, B. R., O'Donnell, M. R., Kim, J. Y. Raz, D. J. (2016). Survival following lung resection in immunocompromised patients with pulmonary invasive fungal infection. Eur J Cardiothorac Surg, 49(1), 314-320. doi: 10.1093/ejcts/ezv026

Yapar, N. (2014). Epidemiology and risk factors for invasive candidiasis. Ther Clin Risk Manag, 10, 95-105. doi: 10.2147/ TCRM.S40160

Yildirmak, M. T., Gedik, H., Simsek, F., Iris, N. E., \&t Gucuyener, A. (2013). Is Timely and Appropriate Antifungal Drug Enough for Survival of Adult Cases with Candidaemia? Five-year Experience. West Indian Med J, 62(8), 724-730. doi: 10.7727/ wimj.2012.140

Zaini, v., Kordbacheh, P., Mahmoudi, M., Safara, M., \&t Shekari, H. (2012). In vitro Susceptibilities of Iranian Clinical nonalbicans Candida species to Caspofungin and Ketoconazole J. Basic. Appl. Sci. Res., 2(2), 1608-1614. 